\title{
DOING ETHICS: A UNIVERSAL TECHNIQUE IN AN ACCESSIBILITY CONTEXT
}

\author{
Christopher R. Simpson ${ }^{1}$, Liddy Nevile ${ }^{2}$, Oliver K. Burmeister ${ }^{3}$ \\ ${ }^{1}$ Adjunct Teaching Fellow, School of Information Technology, \\ Swinburne University of Technology, Australia, \\ E-mail: csimpson@swin.edu.au; \\ ${ }^{2}$ E-mail: Liddy.Nevile@motile.net; \\ ${ }^{3}$ Swinburne Computer-Human Interaction Laboratory, School of Information Technology, \\ Swinburne University of Technology, Australia, \\ E-mail: oburmeister@it.swin.edu.au;
}

\begin{abstract}
Thinking ethically is difficult without a background in moral philosophy. Asking people to embrace ethics without offering practical explanation of the efficacy of so doing, is a thankless task. A technique that people can follow easily, that helps them value ethical behaviour is needed. Illustrations of such a procedure show how professional scenarios are used in the domain of web accessibility. Scenario design and extensions to the technique are considered.
\end{abstract}

Keywords Ethics; Accessibility; Case-studies; Dilemma resolution, IT.

\section{INTRODUCTION}

Whether a student, a retiree or in professional practice, today one confronts many situations where it would be helpful to have a particular way of sifting through issues to determine appropriate courses of action.

Gordon Preece (2002) writing on a recent topical issue put it this way: 'The womb is like an ethical war-zone. Embryonic stem-cell research, deaf lesbians choosing deaf-babies, IVF embryos chosen and conceived to save existing children, single and lesbian women accessing IVF. Hardly a day goes by without a new ethical dilemma. The pace of technological change and precedent makes it almost impossible to keep up. '

This paper gives the background that gave rise to this technique, the process itself, and then makes use of exemplary scenarios to illustrate how the procedure can be useful to practitioners. One scenario illustrates the approach, while a second illustrates the value in designing appropriate scenarios. The paper ends with suggestions of how the technique, first used in a student context, can be extended to suit professionals from different disciplines as multi-disciplinary teams engage in activities such as building websites.

\section{BACKGROUND}

At Swinburne University of Technology, 'Professional Issues in Information Technology' (PIIT) is taught to all final year IT students. It can be taken by students from other faculties as an elective, although students without industrial experience are strongly discouraged from taking the subject. Over the past 12 years, as the subject has evolved, the focus has been to explore real cases that will confront young (and not so young) professionals in the workplace. This is achieved by having about half the lectures led by industry speakers, as well as through tutorial work, debate and discussion that explore cases collected over the years, and draw heavily on the Industry Based Learning (IBL) experiences of the students.

Students have often expressed difficulty in thinking through ethical situations. PIIT is usually the only subject requiring this in their computing courses. Despite their lack of familiarity with this type of work, almost all students, after finishing the subject, rate it as one of the most valuable to their professional development and one of the most enjoyable in their course.

To overcome students' initial difficulties, a simple approach to scenario analysis has been developed. Formative feedback during the semester from tutors has often involved answering student queries in terms of this process, getting them to think through pertinent issues. Similarly, summative assessment has shown that critical thinking and meaningful consideration of multiple alternate solutions to derive a best fit, most frequently occur when this approach is adopted by students.

Although the technique was developed for student use, it is not limited to this context. In the view of the authors, it is widely applicable. To illustrate this point, the scenarios below offer a professional Internet developer's viewpoint, with the technique applied as though carried out by professional peers. 


\section{The doing ethics technique}

The technique of analysis depends upon asking questions. It has been found that the order in which the questions are asked is also important. The following questions, in this order, have been found to work best (through trial and error over many semesters).

Q1 What is going on? - What are the facts?

Q2 What are the issues?

Q3 Who is affected?

Q4 hence, What are the ethical issues and implications?

Q5 What can be done about it? - What options are there? and

Q6 Which option is best? - and Why?

\section{Benefits}

An injunction to 'think ethically' about a situation is not helpful. Perhaps if one has a background in moral philosophy this would work, but usually both students and IT professionals require some form of guidance as to how to achieve an appropriate outcome. The technique has proven itself as a means to achieving this, at least for PIIT students.

This approach is not dependent on a particular standard, such as the code of ethics of a particular professional society. It is a technique that can be applied in a variety of circumstances, not limited by technological, cultural or religious background.

The approach is not limited by one's moral philosophy. One can use this technique effectively and be an objectivist, holding that certain moral truths remain good independently of personal likes and dislikes, or a relativist, holding that truths are relative to the individual or one's culture. Similarly, this approach can be used by consequentialists, holding that consequences determine if something is ethical, and by deontologists, holding that some things, regardless of the consequences, are right or wrong in themselves. The technique is a means of arriving at an ethical outcome appropriate within (or at least independent of) personal belief systems.

\section{Limitations}

The best results obtained in the PIIT classes have been when the technique has been used in tutorial groups, rather than have students submit individual reflections on particular cases. Individual reflection yields significant results and students have voiced their delight at being able to work through difficult situations using this technique, but even so, further insights and better solutions are derived when it is used in a group situation. Solving scenarios collaboratively also adds credibility to the process, in that much of the professional development takes place in multidisciplinary teams. And that creates new a problem - which ethical standard should one adopt? In multidisciplinary teams, professionals often belong to different professional societies, each with similar, but not identical, codes of ethics. In a student context this leads to interesting discussions, in a professional setting it may lead to conflicting standards that are difficult to resolve.

Another limitation is objectivity. One advantage of thinking through cases, such as those below, before being confronted with real situations, is that it becomes easier to be objective. When confronted with an ethical dilemma in real life, subjectivity can prevent one from being able to make the most appropriate judgments. This is where reference to one's peers, co-workers or members of one's professional society can be helpful. Such people can use this technique to work objectively through the issues and advise the person in the situation of the most appropriate course of action.

\section{Illustrating use of the technique}

Educational suppliers, world-wide, are depending upon their belief in the commodification and commercialisation of education to offset the increasing cost of education. Typically, they look for or develop learning object repositories and skeletal structures or 'skins' within which to serve up compilations of bitesized pieces, custom-built for individual needs. This form of education could benefit those with disabilities at least as much, if not more, than others. Such disabilities can take the form of physical or mental human disabilities, or resource or location based incapacities. This is the context of the first case. 


\section{Case 1: Universal access}

Government funding has been given to a university consortium establishing a repository of resources for school teachers. They have engaged you to develop a search facility for teachers who will use it to discover small chunks of useful material, located anywhere. The chunks will be strung together to make customised lessons for students who cannot attend school, for one reason or another, or whose teachers do not include specialists in subjects the students want to study. The client group knows that it is more 'attractive' to their funding agency if there is a lot of multimedia product in the selection. You know that it is hard to produce accessible multimedia and there is hardly any 'out there'. You are asked to advise your client about accessibility.

Q1 What is going on? - What are the facts?

I. I report to the university consortium.

II. I am to develop a repository search facility for school teachers to extract useful teaching materials to develop suitable lessons.

III. Target students are supervised by non-specialists (possibly remotely, at home, or in care).

IV. The government funding agency likes multimedia.

V. Accessible multimedia content is scarce and 'hard' to develop.

VI. The consortium requires 'accessibility' advice.

Q2 What are the issues?

VII. What are the contractual details/arrangements?

VIII. Accessible multimedia may be costly to develop. There appears to be a funding bias towards multimedia content. How much multimedia is envisaged? Would a simple multimedia put funding at risk? Advice to the consortium might be rejected (along with this job) by recommending a simple, 'non-glossy' approach.

IX. What are the exact requirements for the multimedia? What standards are to be applied? What is the budget for it and how much of that is for the extra accessibility work?

X. Sophisticated multimedia is unlikely to be easily accessed/afforded by all supervisors or students due to special needs and telecommunications inequities.

XI. What range of resources do supervisors/teachers and students have ready access to?

XII. What range of disabilities do the target students suffer, if any? How will these be identified and accounted for?

XIII. What level of training and support is envisaged for teachers, non-specialist supervisors and students?

XIV. It is not clear if 'normal' school teachers are to benefit from this system.

Q3 Who is affected?

Adversely...

XV. My team, if our carefully considered recommendations are 'too pedestrian' and so rejected.

XVI. Students with disabilities, if systems are badly designed or too costly.

XVII. All students, if the system is too cumbersome (costly, slow or otherwise hard to use).

XVIII. All students, if the resulting lessons are inadequate, trivialised or unsupported.

XIX. Teachers (particularly non-specialists), without sufficient opportunities (time, funds, help or equipment) to learn about and receive prompt, ongoing support with the system.

XX. All those unjustly excluded from the system, through negligence or economics or whatever.

XXI. The consortium (and every party concerned), if the system fails, for any reason.

Positively...

XXII. Everyone (including the community), if the job is done with transparency and integrity.

Q4 hence, What are the ethical issues and implications?

XXIII. Commitment, integrity and mutual trust between all stakeholders.

XXIV. Clarification of project intentions and removal of political bias by the funding agency and consortium, and transparency in discussions regarding advice supplied to them. 
XXV. Realistic definition of project scope, based upon: professional design, ethical prioritising of need, provision of sufficient ongoing support.

XXVI. Discovery of the real needs of targeted teachers and students.

XXVII. Strenuous avoidance raising false expectations.

XXVIII. Maintenance of sufficient quality of teaching material.

XXIX. Particular attention to support of non-specialist teachers.

XXX. Particular attention to support of students with disabilities.

XXXI. Particular attention to matching technical sophistication with affordability and accessibility.

...Without attention to the above points, the project cannot proceed successfully, many people and reputations will be hurt, and precious funds will be wasted.

Q5 What can be done about it? - What options are there?

XXXII. Advise the client that you need to know more but suspect that this contract will need to specify high standards of accessibility 'in case' there are students or teachers with disabilities as it is almost impossible to make inaccessible content accessible retrospectively.

XXXIII. Advise them to:
A. Undertake extensive feasibility studies and exhaustive prioritisation before development.
B. Identify the primary beneficiaries of the system.
C. Build in mutual safeguards to help alleviate unforseen problems.
D. Ensure the ongoing viability of the resulting resource by having sufficient support services.
E. Avoid over-sophisticated technology unless it is clearly affordable by targeted users and disadvantages nobody.

Q6 Which option is best? - and Why?

XXXIV.In this case, there are still too many unknowns for a decision to be made so the best advice will have to be about what needs to be discovered and how, so that such a decision can be based on informed criteria.

\section{Scenario design}

Case studies have frequently been employed to illustrate ethical principles and particularly conflicts and prioritisations amongst those principles. For instance, Anderson et al. (1993) employ scenarios to explore the application of a new code of ethics in different professional settings. Clement (1993), in writing up experiences in a Computer-Supported Cooperative Work workshop, found that discussing scenarios was a productive means to exploring privacy issues. Similarly, Burmeister (2000) used case studies to illustrate the application of the Australian Computer Society (ACS) Code of Ethics to professional practice.

Why design and explore scenarios? Why not just wait until one is confronted with an ethical dilemma in the workplace and then apply the above technique? There are several reasons. In general terms there is the advantage of using scenarios to explore situations ahead of time, while one can engage objectively in the discussion, without a subjective involvement in a particular situation. More specifically, in the accessibility context, scenarios help to identify one's knowledge. Considering accessibility, there are many factors that should be taken into account, as is seen in the discussion above regarding Case 1 and in the discussion about possible extensions to the technique of ethical analysis that follows. Similarly, in other contexts, scenarios can be employed to explore one's knowledge of a particular domain. By asking people what they would do in a particular situation, one can readily identify the limits of that person's knowledge.

For the purposes of ethical scenario analysis, scenarios should be neither self-evident nor simple. Instead, there should be ambiguities that require moral reflection, leading not always to a 'right' solution, but rather towards a solution that is satisfactory. As stated in the limitations above, further reflection by others should yield alternate and perhaps even better solutions than those suggested by the original participants in the discussion. Similarly for Case 1 above, this process gives rise to possible solutions, it does not necessarily lead to the best or even to a good solution. But it does guide people to consider broad, complex issues in a systematic fashion. It is a useful instrument or framework for ethical decision-making.

Another use of scenarios can be to raise awareness of issues. For instance, with little re-working, the two cases in this paper could be phrased so accessibility issues are inconspicuous. Then, in an audience of web designers, the discussion can be used to raise awareness of accessibility considerations, through simple 'what if' additions ... What if a user was visually impaired? What if a deaf person tried to access the video clip? 


\section{Case 2: Conflict of interest}

Australian government sites were mandated to conform to at least single 'A' level of the World Wide Web Consortium (W3C) Web Accessibility Initiative (WAI) standards, by the end of 2001, seen as best practice in Australia (Sampson-Wild and Burmeister, 2001). Yet the recent call for tenders to audit compliance of government sites, which closed $20^{\text {th }}$ June this year (Innes, 2002), is likely to find very few government sites complying with these standards. The following case assumes your organisation was the successful tenderer ...

Your client is a local government agency. You have been asked to develop an accessibility assurance strategy as part of a preliminary contract. You are keen to pick up the contract to build whatever you convince the government to do about accessibility testing, so you are wondering what to recommend. You realise that the agency wants to be told that its materials are accessible, rather than the converse, so you are wondering how high to set the bar for accessibility. You know there are automatic checks available for all the 508 requirements. You could not afford to offer to do all the manual checks required by the WCAG and anyway, suspect the agency's materials would not look good if tested by WCAG.

Like the earlier case, this one has multiple facets with no easy resolution. Recent scandals in the US have involved large accounting firm practices where auditors also have consulting roles, creating a conflict of interest that has caused various now well-publicised problems. Such conflicts are not peculiar to accounting, as is seen in this case.

As mentioned above, one use of scenario design is to explore the domain knowledge of participants. Both cases above involve the government. This was a deliberate, because it raises questions about the applicability of legislation concerning accessibility. Also, in the $2^{\text {nd }}$ case, are legal implications which can either be teased out by a facilitator or left to arise naturally in discussion by participants querying 'WCAG' or '508'. Thus, there is the possibility to discuss the boundaries between legal and ethical considerations. More is made of this point below.

\section{Extending the technique: from student to practitioner}

The technique began in a student context, but to be more suitable for professionals, it should be extended. IT work today brings together people from different disciplines. This is especially common in complex systems design, typical when building web sites. The multi-disciplinary requirements of professionals might better be met with some additional modifications. The extensions begin to apply at question $3 \ldots$

\section{Q3 Who is affected?}

There are some situations in which, in addition to asking 'who' is affected, it may also be appropriate to ask how the 'environment' is affected. Further, this need not be limited to the 'environment' outdoors, but could also refer to workplace culture, the connectedness of a community, or other situational circumstances.

As stated above, it is significant the government is mentioned as the use of government funding or publication by the government may change the potential liability of the developers and publishers.

Another environmental consideration in Case 1 involves small rural schools that cannot afford specialist teachers, given their small class sizes. In that case, other kinds of accessibility considerations come to the fore, especially in terms of telecommunications access. Low bandwidth might have implications in Case 1 on the type of multimedia design. For instance, high-quality images, ideal for high-speed transmission, might not be appropriate. If a rural student is also disabled, then that further complicates the issues. One frequently employed multimedia technique involves the use of 'chat' environments, yet rural students with disabilities would possibly be totally excluded from participation in such class activities.

Q5 What can be done about it? - What options are there?

This question could be extended to include the following three sub-questions:

I. What would be 'the right thing' to do?

II. Who/what suffers if you do not do the right thing?

III. What additional costs will be incurred if you do the right thing?

Doing 'the right thing' is a principle that is often cited in the US and is akin to 'setting a good example', or observing 'best practice'. 'The right thing' is not always the most economical, the fastest or the easiest thing to do, but it is valued for its ethical advantage. Given the wide acceptance of the right thing in some 
circumstances, it has also come to mean 'that which will have the most beneficial long-term as opposed to immediate effects'.

Q6 Which option is best? - and Why?

This question could be extended to include the following sub-question:

IV. Who gains if you do the right thing?

Political advantage comes in many forms. It does not always coincide with economic or social advantage. Understanding the potential for bias among the stakeholders can help evaluate potential decisions.

$$
\text { V. And Why? }
$$

This inevitably casts one's view into the future, where the consequences of our decision will be thrust upon our successors and children, and their environment. Will our decision be seen as wise and far-sighted?

\section{Multi-disciplinary extensions}

The following two additional questions are inappropriate in the student context, because rarely do IT students have sufficient experience during or prior to their courses to deal adequately with these issues. Typically, the ACS's Code of Ethics requires professionals to consider the legal ramifications of their work on their employer, their clients and the eventual users of the products. But when ACS-accredited professionals work with professionals from other disciplines, they often find that ethical codes of behaviour differ in trivial, or significant, ways and have to make decisions about which 'ethics' to follow. In other contexts, there may be practical considerations to deal with.

The technique could be further extended to include:

VI. What does the law say?

VII. What chance is there it will be enforced?

Particularly in web site development, where cross-border business is concerned, legal compliance becomes a complex issue. The cases above show that compliance in one jurisdiction does not imply one's product complies in another. For instance, there are subtle differences between complying in Australia with the accessibility requirements for Australian government sites, and compliance requirements for the same sites, in the US. In Case 2, mention of particular standards (WCAG and 508) are introduced to lead participants to focus on relevant legislation.

\section{CONCLUSIONS}

Though this technique evolved out of the need to help students think through ethical dilemmas in a systematic way, it is not limited to a student context. The process will work just as well in peer evaluative situations. For instance, one of the limitations referred to above is that when confronted with an ethical dilemma in the workplace, it is difficult to remain objective in one's analysis. In such a situation, this technique will help a knowledgeable peer/co-worker to work objectively through the issues to develop appropriate advice on how best to proceed. Similarly, members of the ACS are encouraged, when confronted with ethical dilemma, to seek help from the society and/or from the Australian Institute of Computer Ethics (AiCE). People who are asked for advice need a way of sifting through the pertinent issues to devise an appropriate response. The ACS Code of Ethics goes part of the way to do this. Yet it really only sets the standard, as it does not provide a way of arriving at the best (or at least a satisfactory) solution. What has been proposed in this paper is a recipe for formulating such a solution.

Further work is needed to validate the technique. At this time, there is only anecdotal evidence from tutors involved in formative and summative assessment of student work, and from students themselves. There is also anecdotal work that supports the value of such scenarios when trying to alert practitioners to the problems of accessibility, and when working towards recommendations for action in response to those who request help from AiCE members. Formal data needs to be gathered as to the efficacy of this technique for isolated practitioners, as well as to enhance it, if possible. 


\section{REFERENCES}

Anderson, R.E., Johnson, D.G., Gotterbarn, D. \& Perrolle, J. (1993) Using the New ACM Code of Ethics in Decision Making, Communications of the ACM, 36(1), pp.98-107.

Burmeister, O. K. (2000), Applying the ACS Code of Ethics, Journal of Research and Practice in Information Technology, 32(2), May, pp.107-120.

Clement, A. (1993): Privacy Considerations in CSCW: Report of the CSCW'92 Workshop, SIGCHI, ACM Press, 25(4), pp.34-39.

Innes, G. (2002) Request for proposals: Web site access survey, HREOC Disability Rights, http://www.humanrights.gov.au/disability_rights/webaccess/survey02_rfp.htm, 18/7/2.

Preece, G. (2002) A war-zone in the womb, The Melbourne Anglican, May, No 393.

Sampson-Wild, G. and Burmeister, O. K. (2001) The Continuing Evolution Of Best Practice Principles In Designing For Web Accessibility, Proceedings OZCHI2001 Usability and usefulness for knowledge economics, Nov, pp.122-127. 